\title{
INDICATRIC TORSION IN A SUBSPACE OF A RIEMANNIAN SPACE
}

T. K. PAN

1. Definition. In a former paper [4] the author defined indicatric torsion of a vector field in a direction to generalize the concept of geodesic torsion of a curve in a surface of a Euclidean three dimensional space. The present note extends the investigation to a subspace of a Riemannian space. Special concern is for hypersurfaces in a Riemannian space and in a Euclidean space.

Let $V_{n}$ be a Riemannian space with fundamental quadratic form

$$
\phi=g_{i j} d x^{i} d x^{j} \quad(i, j=1, \cdots, n)
$$

which is immersed in a Riemannian space $V_{m}$ with fundamental quadratic form

$$
\psi=a_{\alpha \beta} d y^{\alpha} d y^{\beta} \quad(\alpha, \beta=1, \cdots, m) .
$$

Let $V_{n}$ be defined in $V_{m}$ by equations of the form

$$
y^{\alpha}=y^{\alpha}\left(x^{1}, \cdots, x^{n}\right)
$$

where the functional matrix $\|\partial y / \partial x\|$ is of rank $n$.

Let $v: v^{\alpha}=y^{\alpha}, v^{i}$ be a unit vector field in $V_{n}$ and let $C: x^{i}=x^{i}(s)$ be a curve in $V_{n}, s$ being its arc length. We assume $v$ and $C$ to be of class not less than 2 and 3 , respectively. Let $\left.N_{\sigma}\right|^{\alpha}$ for $\sigma=n+1, \cdots, m$ denote $m-n$ mutually orthogonal unit vectors normal to $V_{n}$. Define

$$
\begin{aligned}
\left.\eta_{1}\right|^{\alpha} & =v^{\alpha},\left.\quad \eta_{2}\right|^{\alpha}=\left.{ }_{v} k_{1} N_{\nu}\right|^{\alpha}, \\
\left.\eta_{r+1}\right|^{\alpha} & =\left.\eta_{r}\right|_{; i} ^{\alpha} \frac{d x^{i}}{d s} \quad(r=2, \cdots, m-1)
\end{aligned}
$$

where

$$
{ }_{v} k_{1}=\left.a_{\alpha \beta} N_{\nu}\right|^{\alpha}\left(\frac{\partial^{2} y^{\beta}}{\partial x^{i} \partial x^{j}}+\frac{\partial y^{\gamma}}{\partial x^{i}} \frac{\partial y^{\delta}}{\partial x^{j}} \Gamma^{\beta} \gamma^{\delta}-\frac{\partial y^{\beta}}{\partial x^{k}}\left\{\begin{array}{c}
k \\
i
\end{array}\right\}\right) v^{i} \frac{d x^{j}}{d s},
$$

$\Gamma_{\gamma \delta}^{\beta}=$ Christoffel symbols in $V_{m}$,

$$
\left\{\begin{array}{cc}
k \\
i & j
\end{array}\right\}=\text { Christoffel symbols in } V_{n} \text {. }
$$

If the vectors $\left.\eta_{\delta}\right|^{\alpha}$ for $\delta=1, \cdots, m$ are assumed to be linearly inde-

Presented to the Society, December 29, 1955; received by the editors April 11. 1956. 
pendent, the following linear combinations of them for $p=1, \cdots m$, form a set of $m$ mutually orthogonal vectors [1, pp. 103-104]:

$$
\left.\lambda_{p}\right|^{\alpha}=\left.\left(\frac{b_{p}}{b_{p-1}}\right)^{1 / 2} \eta_{\lambda}\right|^{\alpha} B_{p}^{\lambda} \quad(\lambda, \mu=1, \cdots, p)
$$

where

$$
\begin{aligned}
b_{0} & =1, \quad b_{p}=\left|b^{\mu}{ }_{\lambda}\right|, \\
b^{\lambda}{ }_{\mu} & =b^{\mu}{ }_{\lambda}=a_{\alpha \beta} \eta_{\lambda}\left|{ }^{\alpha} \eta_{\mu}\right|^{\beta}, \\
b^{\mu}{ }_{\lambda} B^{\lambda}{ }_{\rho} & =\delta^{\mu}{ }_{\rho} .
\end{aligned}
$$

The formulas of Frenet for $v$ along $C$ in $V_{m}$ read as follows:

$$
\lambda_{p}\left|{ }_{i i} \frac{d x^{i}}{d s}=-{ }_{v} K_{p-1} \lambda_{p-1}\right|^{\alpha}+\left.{ }_{v} K_{p} \lambda_{p+1}\right|^{\alpha}
$$

where ${ }_{\diamond} K_{p}$ for $p=1, \cdots, m-1$ are, respectively, the associate curvatures of order $1, \cdots, m-1$ of the vector field $v$ for the curve $C$.

From (1.2) we have

$$
\left.\lambda_{2}\right|^{\alpha}=\left.N_{\nu}\right|^{\alpha},
$$

and from (1.3) we have for $p=2$

$$
N_{\nu}\left|\alpha_{; i} \frac{d x^{i}}{d s}=-{ }_{v} K_{1} v^{\alpha}+{ }_{\nu} \tau_{0} \lambda_{3}\right|^{\alpha}
$$

where ${ }_{v} \tau_{g}$ is employed to denote ${ }_{v} K_{2}$ in the case under consideration.

The scalar, ${ }_{v} \tau_{g}$, is called the indicatric torsion of $v$ along $C$ in $V_{n}$ in $V_{m}$ relative to $\left.N_{\nu}\right|^{\alpha}$. When $V_{n}$ is a surface in a Euclidean three dimensional space, ${ }_{v} \tau_{g}$ is the indicatric torsion of $v$ in the direction of $C$ as introduced in [4]. If $v$ is furthermore tangent to $C,{ }_{0} \tau_{0}$ becomes the geodesic torsion of $C$ as defined in classical differential geometry.

2. Formulas. Denote the unit vector tangent to $C$ by $t^{i}$ in $V_{n}$ and by $T^{\alpha}$ in $V_{m}$. Multiplying both sides of (1.4) by $a_{\alpha \beta} \lambda_{3} \mid{ }^{\beta}$ and summing over $\alpha$, we have

$$
{ }_{{ }^{\prime} \tau_{g}}=a_{\alpha \beta} \lambda_{3}\left|\alpha{ }^{\alpha}{ }_{\nu}\right|_{; i} t^{i}=\left.\left.a_{\alpha \beta} \lambda_{3}\right|^{\alpha} N_{\nu}\right|^{\beta}{ }_{, \gamma} T^{\gamma},
$$

that is,

$$
{ }_{v} \tau_{g}={ }_{N} K_{G} \cos \theta
$$

where $\theta$ denotes the angle between $\left.\lambda_{3}\right|^{\alpha}$ and the unit vector $w^{\alpha}$ defined by

$$
N_{\nu} \mid{ }^{\alpha}{ }_{\gamma} T^{\gamma}={ }_{N} K_{G} w^{\alpha}
$$


with ${ }_{N} K_{G} \geqq 0$.

It is seen from (1.4) that the vector $w^{\alpha}$ is linearly dependent on the orthogonal vectors $v^{\alpha}$ and $\left.\lambda_{3}\right|^{\alpha}$. Hence, if ${ }_{N} K_{G} \neq 0$ and if $\phi$ is the angle between $w^{\alpha}$ and $v^{\alpha}$, we have

$$
{ }_{v} \tau_{o}=e_{N} K_{G} \sin \phi
$$

where $e= \pm 1$.

By the help of the following formula $[5$, p. 59]

$$
\sin \tau=\frac{\left(\delta_{a b}^{p q} g_{i p} g_{j q} A^{a} B^{b} A^{i} B^{j}\right)^{1 / 2}}{\left(g_{i j} A^{i} A^{j}\right)^{1 / 2}\left(g_{i j} B^{i} B^{j}\right)^{1 / 2}}
$$

where $\tau$ is the angle between two vectors $A^{i}$ and $B^{i}$ in a Riemannian space with fundamental covariant tensor $g_{i j}$, we have from (2.2) the following relation

$$
\left({ }_{v} \tau_{\theta}\right)^{2}=\left.\left.\delta_{\alpha \beta}^{\lambda \delta} a_{\lambda \gamma} a_{\delta \tau} N_{\nu}\right|_{; i} ^{\alpha} N_{\nu}\right|_{; j} ^{\gamma}{ }_{i j}^{\beta} v^{\tau} t^{i j} j .
$$

With the following definitions

$$
\begin{aligned}
& \left.\Omega_{\nu}\right|_{i j}=\left.y^{\alpha}{ }_{; i j} a_{\alpha \beta} N_{\nu}\right|^{\beta}, \\
& \left.\vartheta_{\mu \nu}\right|_{i}=\left.\left.a_{\alpha \beta} N_{\nu}\right|^{\alpha}{ }_{i i} N_{\mu}\right|^{\beta},
\end{aligned}
$$

it is derived that

$$
\left.N_{\nu}\right|_{i i} ^{\alpha}=-\left.\Omega_{\nu}\right|_{i k} g^{k j} y^{\alpha}, j+\left.\left.\sum_{\mu} \vartheta_{\mu \nu}\right|_{i} N_{\mu}\right|^{\alpha}
$$

Substituting this expression into (2.3) and simplifying, we have

$$
\begin{aligned}
\left({ }_{v} \tau_{0}\right)^{2}= & \left.\left.g^{h k} \Omega_{\nu}\right|_{i k} \Omega_{\nu}\right|_{j h} t^{i} t^{j} \\
& -\left.\left.\Omega_{\nu}\right|_{i k} \Omega_{\nu}\right|_{j h} v^{k} v^{h} t^{i} t^{j}+\left.\left.\delta^{\mu \rho} \vartheta_{\mu \nu}\right|_{i} \vartheta_{\rho \nu}\right|_{j} t^{i} t^{j} .
\end{aligned}
$$

When $m=n+1, V_{n}$ is a hypersurface of the enveloping space $V_{n+1}$. Denote by $\Omega_{i j} d x^{i} d x^{j}$ the second fundamental form for $V_{n}$. Denote by $N^{\alpha}$ the unit vector normal to $V_{n}$. Then (2.4) reduces to

$$
\left({ }_{v} \tau_{g}\right)^{2}=\left(g^{h k}-v^{h} v^{k}\right) \Omega_{i k} \Omega_{j h} t^{i} t^{k} .
$$

If $V_{n+1}$ is a Euclidean space $S_{n+1},(2.5)$ becomes

$$
\left({ }_{v} \tau_{g}\right)^{2}=\left(H_{i j}-\Psi_{i j}\right) t^{i} t^{j}
$$

where $H_{i j}$ is the fundamental tensor of the hyperspherical representation of $V_{n}$ and where $\Psi_{i j}$ is defined by

$$
\Psi_{i j}=\Omega_{i k} \Omega_{j h} v^{k} v^{h} .
$$


When $n=2$, we find from (2.6) by some calculation that for a surface in a Euclidean three dimensional space

$$
\begin{aligned}
\left({ }_{v} \tau_{o}\right)^{2} & =\Omega_{i h} \Omega_{j k} \epsilon^{a h} \epsilon^{b k} t^{i} t^{j_{v} p^{q}}\left(g_{a b} g_{p q}-\epsilon_{p a} \epsilon_{q b}\right) \\
& =g_{a p} g_{b q} \Omega_{i h} \Omega_{j k} \epsilon^{a h} \epsilon^{b k} t^{i} t_{v^{p} v^{q}}
\end{aligned}
$$

which implies

$$
{ }_{v} \tau_{o}=e \epsilon^{a h} g_{a p} \Omega_{i h} v^{p} t^{i}
$$

as obtained in $[4]$.

3. Theorems. From (2.1) it is evident that ${ }_{v} \tau_{0}$ is zero if and only if ${ }_{N} K_{G}=0$ or $\cos \theta=0$. The first case means that the geodesic curvature of $\left.N_{\nu}\right|^{\alpha}$ along $C$ in $V_{m}$ is zero; while the second implies that $w^{\alpha}$ and $v^{\alpha}$ are coincident, which case arises if $v^{\alpha}$ is tangent to $C$ and $C$ is a line of curvature of $V_{n}$ in $V_{m}$ corresponding to $\left.N_{\nu}\right|^{\alpha}$. Hence we have

Theorem 3.1. The indicatric torsion of any unit vector field in $V_{n}$ along a curve $C$ in $V_{n}$ relative to a unit vector normal to $V_{n}$ in $V_{m}$ is zero if and only if the unit normal vector moves parallelly along the curve in $V_{m}$ in the sense of Levi-Civita. The indicatric torsion of a unit principal vector field relative to a unit vector normal to $V_{n}$ in $V_{m}$ along the corresponding line of curvature of $V_{n}$ is equal to zero.

It is shown that in $V_{n}$ in $S_{n+1}$ the principal curvature of $t^{i}$ is equal to $\left(H_{i j} t^{i} t^{j}\right)^{1 / 2}$ and that the normal curvature of $v^{i}$ with respect to $C$ is equal to $e\left(\Psi_{i j} t^{i} t^{j}\right)^{1 / 2},[3$, p. $462 ; 2$, p. 963]. Hence we have from (2.6)

THEOREM 3.2. The square of the indicatric torsion of a unit vector field along a curve in $V_{n}$ in $S_{n+1}$ is equal to the difference between the square of the principal curvature of the curve and the square of the normal curvature of the vector field with respect to the curve.

When $C$ is the curve of $v$, the normal curvature of $v$ with respect to $C$ is the normal curvature of $C$. In this case the above theorem reads as

THEOREM 3.3. The square of the indicatric torsion of a curve in $V_{n}$ in $S_{n+1}$ is equal to the difference between the square of its principal curvature and the square of its normal curvature.

When $C$ is the asymptotic line of $v$, the indicatric torsion along the curve of $v$ of the unit vector field formed by those vectors tangent to $C$ is given from (2.6) by

$$
\left({ }_{v} \tau_{0}\right)^{2}=H_{i, v^{v} v^{i}}
$$

Hence we have 
THEOREM 3.4. The indicatric torsion of the unit asymptotic vector field of a unit vector field along the curve of the latter in $V_{n}$ in $S_{n+1}$ is numerically equal to the principal curvature of the latter.

It is obvious from (1.4) that the geodesic torsion $\tau_{0}$ of a curve on a surface in a Euclidean three dimensional space is equal to the product of the magnitude of $\xi_{3}$ and sine of the angle between $\xi_{1}$ and $\xi_{3}$. Hence we get a short way of deriving the following formula

$$
\begin{aligned}
\tau_{\boldsymbol{g}} & =-\epsilon_{i j} d_{k h} g^{h j} t^{i} t^{k} \\
& =-\epsilon^{h l} g_{i l} d_{k h} t^{i} t^{k} .
\end{aligned}
$$

Let $\eta^{i}$ denote the unit vectors tangent to the orthogonal trajectories of $C$, that is,

$$
\eta^{j}=e \epsilon^{j h} g_{i h} t^{i} .
$$

The above formula reduces to

$$
\tau_{o}=e d_{k j} t^{k} \eta^{j}
$$

which gives a new geometric interpretation of the geodesic torsion of a curve as follows:

THEOREM 3.5. The geodesic torsion of a curve at a point on a surface in a Euclidean three dimensional space is numerically the normal curvature of the curve with respect to its orthogonal trajectory at the point.

\section{REFERENCES}

1. L. P. Eisenhart, Riemannian geometry, Princeton, Princeton University Press, 1949.

2. T. K. Pan, Normal curvature of a vector field, Amer. J. Math. vol. 74 (1952) pp. 955-966.

3. - The spherical curvature of a hypersurface in Euclidean space, Pacific J. Math. vol. 3 (1953) pp. 461-466. 457.

4. —_, Torsion of a vector field, Proc. Amer. Math. Soc. vol. 7 (1956) pp. 449-

5. O. Veblen, Invariants of quadratic forms, Cambridge, Cambridge University Press, 1952.

UNIVERSITY OF OKLAHOMA 